\title{
RACK1 promotes prostate cancer cell proliferation, invasion and metastasis
}

\author{
FANGRONG SHEN $^{1}$, CHUNYIN YAN $^{1}$, MING LIU $^{2}$, YAHONG FENG $^{2}$ and YOUGUO CHEN ${ }^{3}$ \\ ${ }^{1}$ Department of Urology, The First Affiliated Hospital of Soochow University, Suzhou, Jiangsu 215006; \\ ${ }^{2}$ Department of Obstetrics and Gynecology, Suzhou Kowloon Hospital, Suzhou, Jiangsu 215021; ${ }^{3}$ Department of Obstetrics \\ and Gynecology, The First Affiliated Hospital of Soochow University, Suzhou, Jiangsu 215006, P.R. China
}

Received February 7, 2013; Accepted June 19, 2013

DOI: $10.3892 / \mathrm{mmr} .2013 .1612$

\begin{abstract}
The aim of the present study was to investigate the functions of RACK1 and its involvement in mechanisms of prostate cancer (PC) cell proliferation, invasion and metastasis. The proliferation, invasion and metastasis of stably transfected DU145 cells with RACK1 was evaluated in vitro as well as in vivo following the establishment of nude mouse models. The expression of Ki67, RACK1, PTEN and androgen receptor (AR) in PC was detected by immunohistochemical analysis. Our results indicated that RACK1 promotes PC cell proliferation, invasion and metastasis in vitro and in vivo. However, knockdown of RACK1 by siRNA in vitro inhibited PC cell proliferation, migration and invasion. PTEN downregulation and Ki67 upregulation were also altered with the upregulation of RACK1; RACK1 staining was strongly correlated with PTEN downregulation and Ki67 upregulation. These data demonstrated that increased RACK1 expression is important in promoting $\mathrm{PC}$ cell proliferation, invasion and metastasis in vitro and in vivo.
\end{abstract}

\section{Introduction}

Prostate cancer (PC) in developed countries accounts for $\sim 15.3 \%$ of all male malignancies and it is the most common non-dermatological epithelial malignant tumor in males (1).

The aggressive nature of cancer cells has been investigated through the analysis of complex signaling pathways that regulate important functions, including cell survival, proliferation, invasion and migration. Cancer cell migration and proliferation are essential factors in invasion and metastasis $(2,3)$. Phosphatidylinositol 3-kinases (PI3Ks) have been shown to be involved in cell processes responsible for malignant behaviors, including survival, proliferation, transformation and adherence,

Correspondence to: Dr Youguo Chen, Department of Obstetrics and Gynecology, The First Affiliated Hospital of Soochow University, 118 Shizi Street, Suzhou, Jiangsu 215006, P.R. China E-mail: chenyouguo@suda.edu.cn

Key words: receptor for activated protein kinase $\mathrm{C} 1$, prostate cancer, proliferation, invasion, metastasis and have been implicated in the carcinogenic mechanisms of cancer $(4,5)$. The PI3K pathway has also been shown to act through its downstream AKT (protein kinase B) molecule by regulating various cell functions, including cell transformation, proliferation, apoptosis, angiogenesis and tumor growth.

PIK3CA is mapped to $3 \mathrm{q} 26$, an area significantly amplified in other types of human cancer, including ovarian, breast, cervical, head and neck and urinary tract cancer $(6,7)$. PIK3CA mutations have been recently identified in several types of human cancer. Moreover, PIK3CA has been found to be mutated in $25,32,27$ and $4 \%$ of brain, colon, lung and gastric cancer, respectively $(8,9)$.

To elucidate the oncogenic mechanisms underlying the development of cancer, we screened a human mammary cDNA library to identify novel PI3K p $110 \alpha$-interacting proteins and to further investigate the detailed mechanisms and functions involved in the metastasis and proliferation of carcinoma. Among the candidate genes, we identified receptor for activated protein kinase C1 (RACK1) as a putative target in human breast carcinoma cells. RACK1, a homolog of the $\beta$-subunit of heterotrimeric $\mathrm{G}$ proteins (10), is involved in the membrane anchoring of multiple proteins (including protein kinase $\mathrm{C}, \mathrm{PKC}$ ) and the coordination of cell adhesion, movement, growth and division $(11,12)$. However, the detailed mechanisms and functions of RACK1 in the metastasis and proliferation of PC cells have yet to be fully elucidated.

The aim of the present study was to investigate the functions of RACK1 and its involvement in mechanisms of PC cell proliferation, invasion and metastasis. Therefore, we investigated the potential downstream signaling pathways following targeted manipulation of RACK1 in the PC cell line DU145. Our data indicated that RACK1 promotes PC cell proliferation, metastasis and invasion in vitro and in vivo.

\section{Materials and methods}

Cells. DU145 cells stably transfected with RACK1 were obtained from the Shanghai Medical College of Fudan University (Shanghai, China).

Cell culture and transfection. Prostate carcinoma DU145 cells were used in the present study. The cells were cultured in DMEM supplemented with $10 \%$ fetal bovine serum (FBS; Gibco-BRL, 
Grand Island, NY, USA), $50 \mathrm{U} / \mathrm{ml}$ streptomycin, $50 \mathrm{U} / \mathrm{ml}$ penicillin, $2 \mathrm{mmol} / 1$ glutamine and maintained at $37^{\circ} \mathrm{C}$. Transient transfection of cells was achieved using Lipofectamine ${ }^{\mathrm{TM}} 2000$ reagent (Invitrogen Life Technologies, Carlsbad, CA, USA). DU145 cells were transfected $12 \mathrm{~h}$ after plating $\left(1.5 \times 10^{6}-2 \times 10^{7}\right.$ cells/dish) according to the transfection protocol, and harvested for investigation within $72 \mathrm{~h}$ after transfection. DU145 cells stably expressing RACK1 were generated by transfection and selection with concentration of geneticin selective G418 antibiotic (Gibco-BRL) for 5-6 weeks at Shanghai Medical College of Fudan University. Surviving DU145 cells were maintained in $1.0 \mathrm{mg} / \mathrm{ml}$ of G418. siRNAs targeting the human RACK1 (GNB2L1) gene sequence, 50CAGATTGTCTCTGGATCTCGA, were obtained from Qiagen (Shanghai, China).

Immunoprecipitation and immunoblotting. Forty-eight hours after transfection, DU145 cells were collected, washed with phosphate-buffered saline (PBS; pH 7.4), and lysed in modified RIPA buffer [50 mM Tris (pH 7.8), 5 mM EDTA, $15 \mathrm{mM}$ $\mathrm{MgCl}_{2}, 150 \mathrm{mM} \mathrm{NaCl}, 1 \% \mathrm{NP}-40,1 \mathrm{mM}$ DTT, $0.5 \%$ sodium deoxycholate and $20 \mathrm{mM} \mathrm{N}$-ethylmaleimide] supplemented with 1 tablet $/ 50 \mathrm{ml}$ of Complete Protease Inhibitor Cocktail (Roche Molecular Biochemicals, Indianapolis, IN, USA). Lysates were cleared by centrifugation $\left(10^{4} \mathrm{x} \mathrm{g}\right.$ for $15 \mathrm{~min}$ at $4^{\circ} \mathrm{C}$ ) and incubated on ice for $2 \mathrm{~h}$ with $2 \mu \mathrm{g}$ of the appropriate antibodies (Abs; anti-Flag from Sigma-Aldrich, St. Louis, MO, USA; anti-GFP from Roche Applied Science, Indianapolis, IN, USA; anti-RACK1 and anti-PI3K p110 $\alpha$ from BD Biosciences, San Jose, CA, USA). Subsequently, $20 \mu$ l of slurry protein A/G Plus Agarose immunoprecipitation reagent (Santa Cruz Biotechnology, Inc., Santa Cruz, CA, USA) was added to each lysate, and incubation was continued with rotation for $\geq 2 \mathrm{~h}$ at $4^{\circ} \mathrm{C}$. The beads were retrieved by centrifugation and washed (by vortex and short spin) 4 times with RIPA buffer and once with PBS. Proteins bound to the beads were eluted by boiling in a 29X electrophoresis sample buffer, separated by SDS-PAGE, and electrotransferred onto PVDF membranes (Millipore, Bedford, MA, USA). After blocking in 5\% nonfat milk, western blot analysis was performed with the indicated primary Abs for $1 \mathrm{~h}$ at room temperature, followed by incubation with horseradish peroxidase (HRP)-conjugated secondary Abs for $1 \mathrm{~h}$ at room temperature. All of the blots were developed using the ECL Plus western blot detection system (Pierce Biotechnology, Inc., Rockford, IL, USA) and X-ray film (Kodak, Rochester, NY, USA). Gel-Pro Analyzer software (version 4.0) was used for the densitometric analysis of each film. The primary Abs used in western blot analysis were the following: phospho-p44/42 MAPK (Thr202/Tyr204), phospho-AKT (Ser473; Cell Signaling Technology, Inc., Danvers, MA, USA); p21, p27, cyclin D1 and D3 (Santa Cruz Biotechnology, Inc.); p53 (Sigma-Aldrich); and CD147 (Novocastra, Newcastle-upon-Tyne, UK). The secondary Abs used for immunodetection were the following: HRP-conjugated goat anti-mouse IgG and goat anti-rabbit IgG (Amersham Biosciences, Uppsala, Sweden). The potent inhibitor of PI3K was purchased from Cell Signaling Technology, Inc.

Immunofluorescence. DU145 cells seeded on 6-well chamber slides were fixed in $4 \%$ paraformaldehyde, blocked in $5 \%$ bovine serum albumin (BSA) and permeabilized in $0.1 \%$
Triton X-100. PI3K p110 $\alpha$ levels were determined with anti-PI3K p110 $\alpha$ (BD Biosciences), followed by a 45 -min incubation with $\mathrm{Cy} 3$-conjugated anti-mouse secondary Ab (Amersham Biosciences). The coverslips were washed, mounted in PBS containing 50\% glycerol, and observed under a confocal laser microscope (Leica Microsystems, Buffalo Grove, IL, USA) or an automated microscope (Leica DMRXA2) equipped with Photometrics Cool SnapES N\&B camera driven by MetaMorph software (Universal Imaging Corporation, Downingtown, PA, USA).

PC cell proliferation assay. PC DU145 cell proliferation was investigated using an MTT cell proliferation kit (Roche Applied Science). DU145 cells were cultured in 96-well plates at a density of $1.0 \times 10^{4}$ cells/well. On 1-6 days following transfection with RACK1 siRNA, DU145 cells were incubated with $10 \mu \mathrm{l}$ of MTT labeling reagent for $4 \mathrm{~h}$, followed by the addition of $100 \mu \mathrm{l}$ of solubilization solution into each well. The plates were maintained in the dark overnight, and the optical density (OD) of each sample was measured at a wavelength of $490 \mathrm{~nm}$ using an ELISA multi-well spectrophotometer (Molecular Devices, Sunnyvale, CA, USA).

In order to determine the DNA content, DU145 cells were harvested by trypsinization $24 \mathrm{~h}$ after transfection, fixed overnight at $4{ }^{\circ} \mathrm{C}$ with $75 \%(\mathrm{v} / \mathrm{v})$ ethanol, washed and incubated in PBS containing $100 \mu \mathrm{g} / \mathrm{ml} \mathrm{RNase}$ and $10 \mu \mathrm{g} / \mathrm{ml}$ propidium iodide (PI) for $1 \mathrm{~h}$ at $37^{\circ} \mathrm{C}$. Data were obtained by a Fluorescence-activated cell sorter (FACS) Canto flow cytometer and analyzed using the FACSDiva software package (BD Biosciences). A minimum of $1 \times 10^{4}$ cells were measured per sample. Gates were based on forward and side scatter set to eliminate cell clusters and cellular debris.

Invasion and migration assay. Scratch assay was performed with $1 \times 10^{6}$ DU145 cells seeded onto a 6-well cell plate, which were allowed to reach full confluence. The monolayer was wounded with a $100-\mu 1$ pipette tip, followed by further culture in the appropriate media as previously described. Images were captured at 0,12, 24 and $48 \mathrm{~h}$. A modified Boyden dual chamber assay was used to measure the directional migration of DU145 cells. In brief, $5 \times 10^{4}$ cells were suspended in serum-free media and added to the upper chamber of an $8-\mu \mathrm{m}$ pore cell culture insert (Becton-Dickinson, San Jose, CA, USA). Serum-containing medium was used as a chemoattractant in the lower chamber. After a 24- to 72 -h incubation at $37^{\circ} \mathrm{C}$ in $5 \% \mathrm{CO}_{2}$, the media and cells that were still in the upper chamber were removed using a cotton swab. The insert was fixed in ethanol and stained using hematoxylin and eosin (H\&E). DU145 cells that were attached to the lower side of the insert were observed using an inverted microscope (Nikon, Tokyo, Japan), and the number of invading DU145 cells was determined by counting the cells in 5 random high-power fields and calculating the mean number of invading cells. We then investigated the ability of the DU145 cells to invade through growth factor reduced Matrigel (BD Biosciences). The Rho kinase (ROCK) inhibitor Y-27632 was purchased from Calbiochem (Darmstadt, Germany). All of the experiments were performed in triplicate.

Zymographic analysis. Matrix metalloproteinase (MMP)-9 and -2 enzymatic activity was assessed using gelatin zymog- 
raphy. Conditioned media from DU145 cells (cultured in serum-free medium for $48 \mathrm{~h}$ ) were collected and concentrated 20-fold by a Centriprep YM-30 device (Millipore, Bedford, MA, USA). Samples were then mixed with Laemmli loading buffer and electrophoresed on a gelatin containing $8 \%$ SDS-PAGE. Following electrophoresis, the gel was washed twice with washing buffer $(100 \mathrm{mM} \mathrm{NaCl}, 2.5 \%$ Triton X-100 and $50 \mathrm{mM}$ Tris- $\mathrm{HCl}, \mathrm{pH} 7.5$ ), followed by a brief rinse in washing buffer without Triton X-100. The gel was then placed with incubation buffer ( $50 \mathrm{mM} \mathrm{NaCl}, 50 \mathrm{mM}$ Tris- $\mathrm{HCl}, \mathrm{pH} 7.5$, $1 \mu \mathrm{M} \mathrm{ZnCl}_{2}, 10 \mathrm{mM} \mathrm{CaCl}_{2}, 0.02 \% \mathrm{NaN}_{3}$ ) at $37^{\circ} \mathrm{C}$ for $24 \mathrm{~h}$. After incubation, the gel was stained with Coomassie Blue R-250 and destained with destaining solution. A clear zone of gelatin digestion represented the MMP activity.

Establishment of the PC nude mouse model. Male nu/nu nude mice (4-6 weeks old) were injected with no-free DU145 control cells (group 1; parental MCF7 cells and cells transfected with empty vector, 5 mice each), M-R4 cells (group 2), T-47D control (group 3) or T-R2 cells (group 4). Each group contained 10 mice. The cells were injected into the second mammary fat pad from the flank $\left(29 \times 10^{6}\right.$ cells in $100 \mu 1$ of PBS). Tumor growth was externally monitored using Vernier Calipers for 4-6 weeks. At 6 weeks after injection (when the mice had not yet died, but some appeared to be sick), all of the mice were sacrificed, and the tumors were removed and fixed in $10 \%$ formalin after weighing and measuring. Tumor volume was calculated using the formula: Tumor volume $\left(\mathrm{mm}^{3}\right)=0.5 \mathrm{a} \mathrm{x} \mathrm{b}^{2}$, where 0.5 is a constant to calculate the volume of an ellipsoid, $a$ is the longest diameter and $b$ is the shortest diameter. Histopathological analysis was performed according to the procedures described below.

Immunohistochemistry. Analysis of RACK1 expression in PC tissue was performed using the RACK1 mouse monoclonal $\mathrm{Ab}$ (LifeSpan Biosciences, Inc., Seattle, WA, USA). For the analysis of Ki67, PTEN, androgen receptor (AR) and MMP2 expression, the corresponding mouse monoclonal Abs from Sigma-Aldrich were used. The positive controls for RACK1 were used with sections of formalin-fixed, paraffin-embedded human adrenal samples as indicated in the instruction manual. The negative controls were incubated with immunoglobulin fraction in place of the polyclonal primary $\mathrm{Ab}$ in the positive tissues mentioned above. The saturation and intensity of the immunostained cells were evaluated over 3 visual fields, at a magnification of x200 under a light microscope (Carl Zeiss, Gottingen, Germany). For the statistical analysis, according to Han et al (13) and our previous studies (unpublished data), the total staining of RACK1 was scored based on the intensity and percentage of cells with RACK1 cytoplasm staining on the following scoring system: score 0 , negative/weak staining for all tumor cells or moderate staining $<30 \%$; score 1 , moderate staining in $>30 \%$ but $<70 \%$ of tumor cells or strong staining within $30 \%$ of tumor cells; score 2 , moderate staining in $>70 \%$, or strong staining in $>30 \%$ of all tumor cells.

Statistical analysis. All data were representative of $\geq 3$ independent experiments with similar results. The results are expressed as the mean \pm SEM from multiple experiments. The Student's t-test was used to determine significant differences (two-tailed, $\mathrm{P}<0.05$ ). The software SPSS 15.0 for Windows (SPSS Inc., Chicago, IL, USA) was used for statistical analyses. $\mathrm{P}<0.05$ was considered to indicate a statistically significant difference.

\section{Results}

Promotion of proliferation, migration and invasion by RACK1 in vitro. To investigate the importance of RACK1 in PC tumorigenesis and progression, we first assessed RACK1 expression in the PC cell line DU145 as mentioned in the Materials and methods section. As shown in Fig. 1A, the cells with a superior invasive ability had a higher RACK1 expression, while the cells with an inferior invasive ability (non-transfected cells) had a lower RACK1 expression.

To detect the effect of RACK1, we initially constructed two stably transfected cell lines: DU145 cells transfected with a RACK1-expressing plasmid or an empty plasmid (control cells) and stable cell lines were established as described in the Materials and methods. The stably transfected clones were obtained and further measured. The RACK1 expression level was increased $350 \%$ in clone DU145 cells and $<200 \%$ compared with the control cells (Fig. 1A). The DU145 cell clones were stable in culture and maintained a high RACK1 level for $\geq 22$ passages in medium containing the selection agent G418. Similar passages of these clones were used in all of the subsequent studies.

In order to examine the effect of RACK1 on the tumorigenic properties of PC cells, we then investigated the in vitro proliferation of stably transfected clones (DU145 cells). Significantly decreased cell doubling times were observed in the RACK1-transfected clones compared with the control cells; the mean doubling times $(95 \% \mathrm{CI})$ were as follows: control, $26.9 \mathrm{~h}$ (range, 26.4-27.4 h), $\mathrm{P}=0.07$ and clone DU145 cells, $19.4 \mathrm{~h}$ (range, 19.1-19.7 h), P<0.001 (Fig. 1A and B). Flow cytometric analysis of clone DU145 cells also demonstrated the ability of RACK1 to promote PC cell proliferation without affecting the rate of apoptosis (Fig. 1B).

Furthermore, the effects of RACK1 on PC cell invasion and migration were determined through scratch tests (Fig. 2A) and Transwell assays without (Fig. 2B) or with Matrigel (Fig. 2C). As shown in Fig. 2, upregulation of RACK1 in clone DU145 cells induced an increased cell migration capacity (Fig. 2B). In addition, DU145 cell invasion abilities were significantly increased, as assessed after $48 \mathrm{~h}$ using modified Boyden chamber assays. The mean number of migrated cells/field (95\% CI) was as follows: clone RACK1 siRNA-transfected DU145 cells, 60 (range, 50-78; $\mathrm{P}<0.001$; Fig. 2C).

To verify the effects of RACK1, we introduced siRNAs specifically targeting RACK1 into the PC DU145 cells and the MTT assay was repeated (Fig. 1B). We next compared the migration and invasion properties of RACK1 knockdown cells compared with that of parental cells (Fig. 2A and B). These data confirmed the promotion of cell proliferation, migration and invasion by RACK1 in PC cells.

Promotion of tumor growth, infiltration and metastasis by RACK1 in a nude mouse model. We investigated the effects of RACK1 on tumor growth and metastasis in an orthotopic nude male primary androgen ablation therapy mouse 

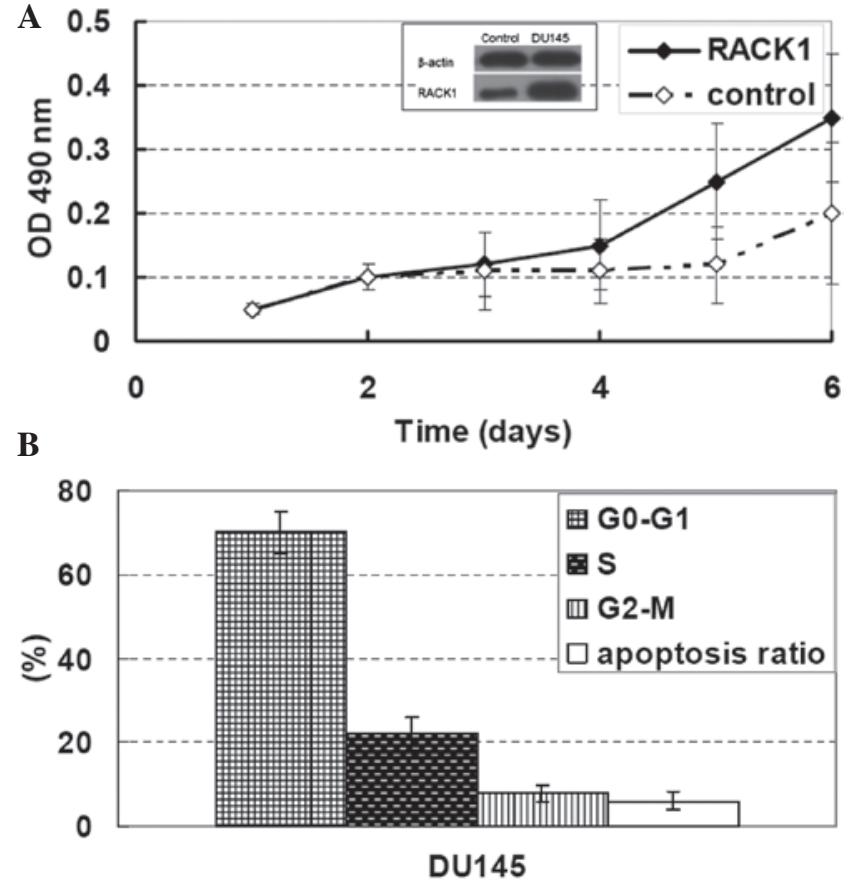

Figure 1. RACK1 upregulation promotes PC cell proliferation. (A) The effect of RACK1 expression on PC cells with invasion potentials is shown. Increased RACK1 expression in RACK1 siRNA-transfected DU145 cells was confirmed using western blot analysis. $\beta$-actin was used as a loading control. MTT assay was used to assess the proliferation of DU145 cells compared with control cells, as well as the effect of RACK1 siRNA on cell proliferation. (B) Flow cytometry showed increased DU145 cell proliferation, while no changes were observed in the rate of apoptosis. PC, prostate cancer.

model. Stably transfected PC DU145 cells, DU145 cells and AR-overexpressing endothelial cells and control cells were implanted into the head and neck from the flank of each male nude mouse as described in Materials and methods. Six weeks following injection, the nude mice were sacrificed, and tumor incidence and weight were determined (Fig. 3). All of the mice $(100 \%)$ that were orthotopically injected with parental cells (control groups) and transfected cells developed tumors. Mice implanted with RACK1-transfected clones or AR- and RACK1-overexpressing cells developed significantly larger tumors compared with the control groups (Fig. 3A-C). A mouse in Group 2 and Group 3, bearing the largest tumor, was found to have a necrotic lower limb that may have been caused by a tumor embolus (Fig. 3A). All of the mice (10/10) in Groups 2 and 3 were observed to have cell infiltration into adjacent tissue (Fig. 3D) and had macroscopically visible metastasis in the liver (one mouse) and/or microscopic metastases in the liver (all 10 mice; Fig. 3E). The expression of Ki67, PTEN and AR was also evaluated in tumor tissue removed from the nude mice (Fig. 4) and suggested an important role for RACK1 in $\mathrm{PC}$ cell growth and metastasis in vivo.

\section{Discussion}

PI3K has been shown to be involved in cell survival, proliferation, cytoskeletal reorganization, metabolism and membrane trafficking $(3,4)$, which are highly implicated in mechanisms of prostate carcinogenesis. In the present study, a human PC cDNA library was initially screened with
A

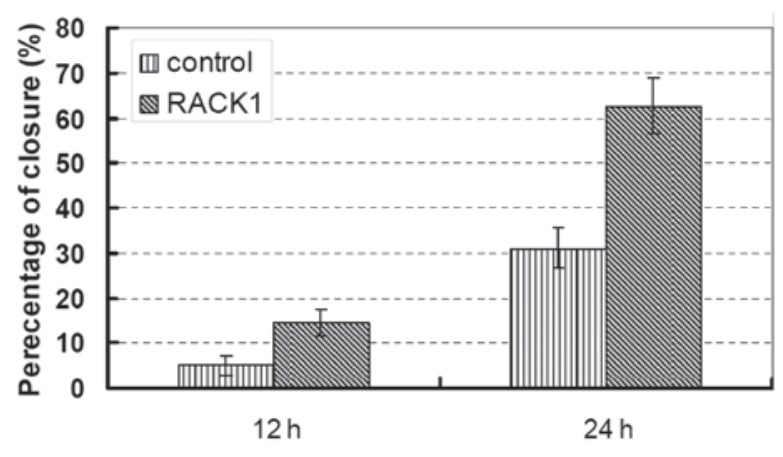

B

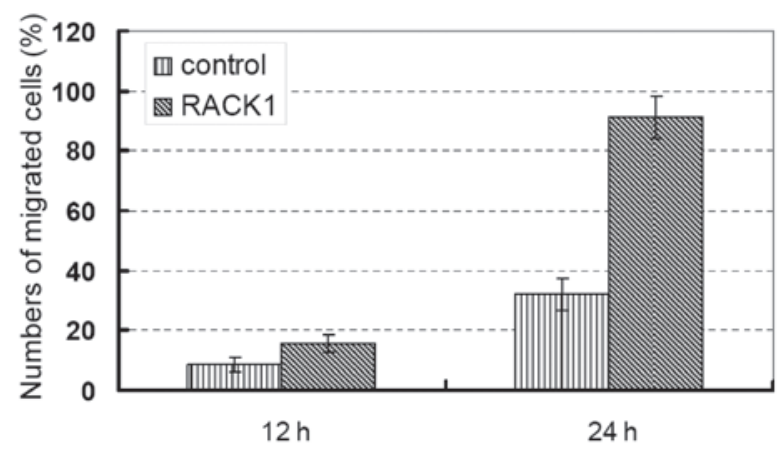

C

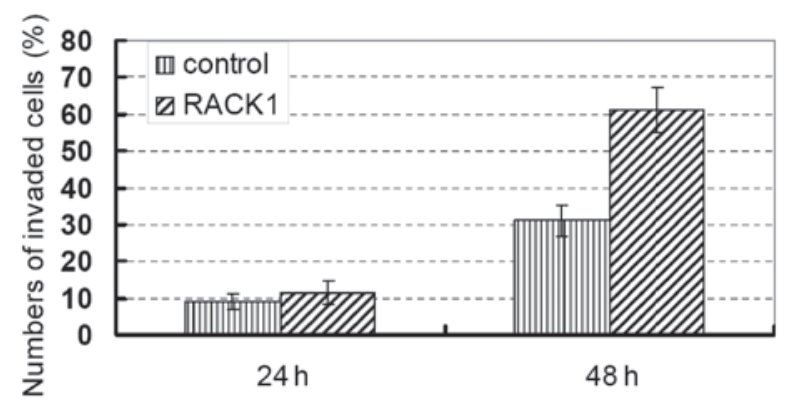

D

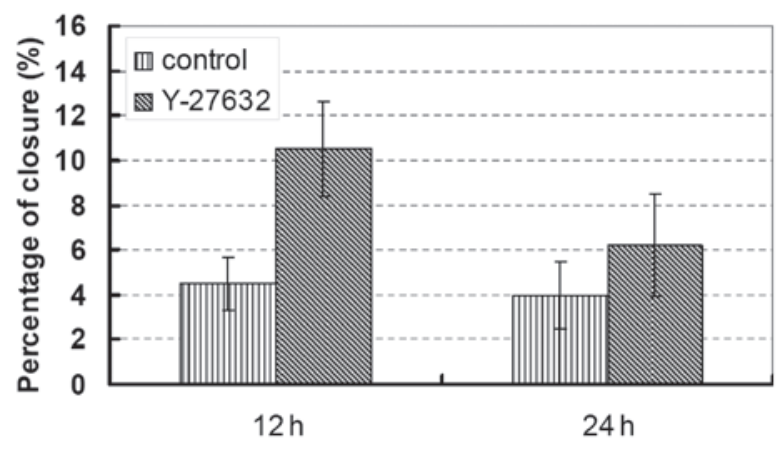

Figure 2. Increased RACK1 expression promotes migration and invasion in PC cells. (A) The migration assay [(B) with modified Boyden chamber] and verification of increased migration of DU145 cells using RACK1 siRNA. (C) Invasion assay utilized Transwell chambers with Matrigel to assess the invasive ability of DU145 cells. (D) Increase in migration caused by RACK1 in PC clone DU145 cells was significantly inhibited after the administration of ROCK inhibitor Y-27632 $(10 \mu \mathrm{M})$. PC, prostate cancer.

PI3K p1 $10 \alpha$ as bait, to identify new mechanisms involved in PC cell growth and progression. Of the proteins identified, RACK1 was selected for detailed analysis. For confirmation, we investigated the PI3K p110 $\alpha /$ RACK1 interaction using in vivo binding experiments. Furthermore, the physiological interaction between PI3K p110 $\alpha$ and RACK1 was confirmed by immunoprecipitation and immunofluorescence analysis of DU145 cells, where RACK1 was identified as a novel 

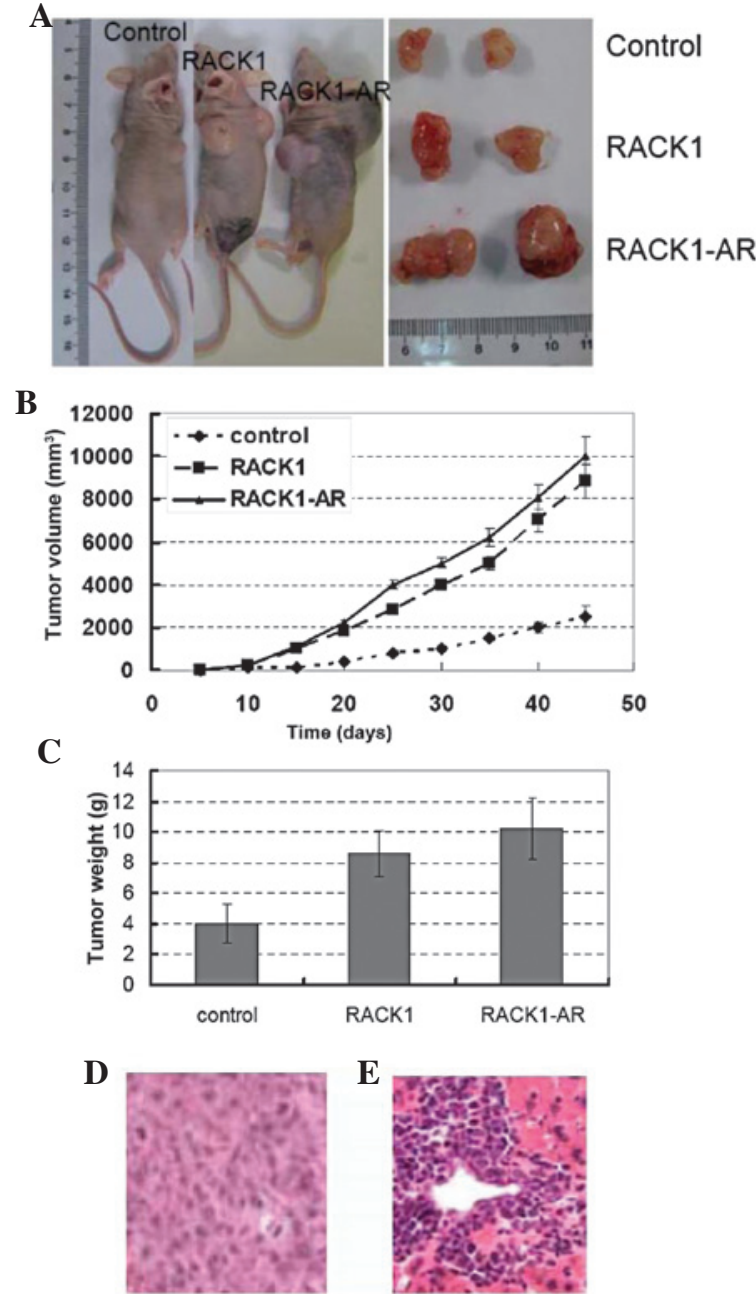

Figure 3. Increased RACK1 expression induced tumor growth and metastasis in a nude mouse model. (A) Representative images of female nu/ nu nude mice 4-6 weeks old that were injected in their second mammary fat pads with PC control cells or RACK1-overexpressing DU145 cells Magnified image of the head neck region shows that the region may have been caused by a tumor embolus induced by the RACK1-overexpressing or AR- and RACK1-overexpressing DU145 cells. (B) Increased tumor volumes of nude mice injected with RACK1-overexpressing or AR- and RACK1overexpressing DU145 cells compared with control cells (parental cells with control vector). Each data point represents the mean value ( \pm SEM) of 4-6 primary tumors. (C) Increased tumor weight of nude mice injected with RACK1-overexpressing or AR- and RACK1-overexpressing DU145 cells compared with control cells (parental cells with control vector). Each data point represents the mean value ( \pm SEM) of $4-6$ primary tumors. Tumor tissues removed from nude mice demonstrated that the RACK1-upregulated group (DU145 cells) developed significantly larger tumors compared with the control groups. Representative images of $\mathrm{H} \& \mathrm{E}$ staining of (D) cell infiltration to adjacent interstitial tissues and skin, and (E) H\&E staining of a representative liver metastasis are shown. PC, prostate cancer; H\&E, hematoxylin and eosin (magnification, x200).

binding partner of PI3K p110 $\alpha$. A series of in vitro and in vivo experiments were performed to investigate the characteristics of RACK1 and to elucidate its role in prostate tumorigenesis. The mechanisms underlying the effect of RACK1 on prostate tumorigenesis were also investigated. The upregulation of RACK1 was shown to significantly promote prostate tumor growth in vitro, using the DU145 cell line by MTT assay and flow cytometry, and in vivo using an orthotopic nude mouse model. Notably, our results are in agreement with those obtained by Wang et al (14), where overexpression of RACK1

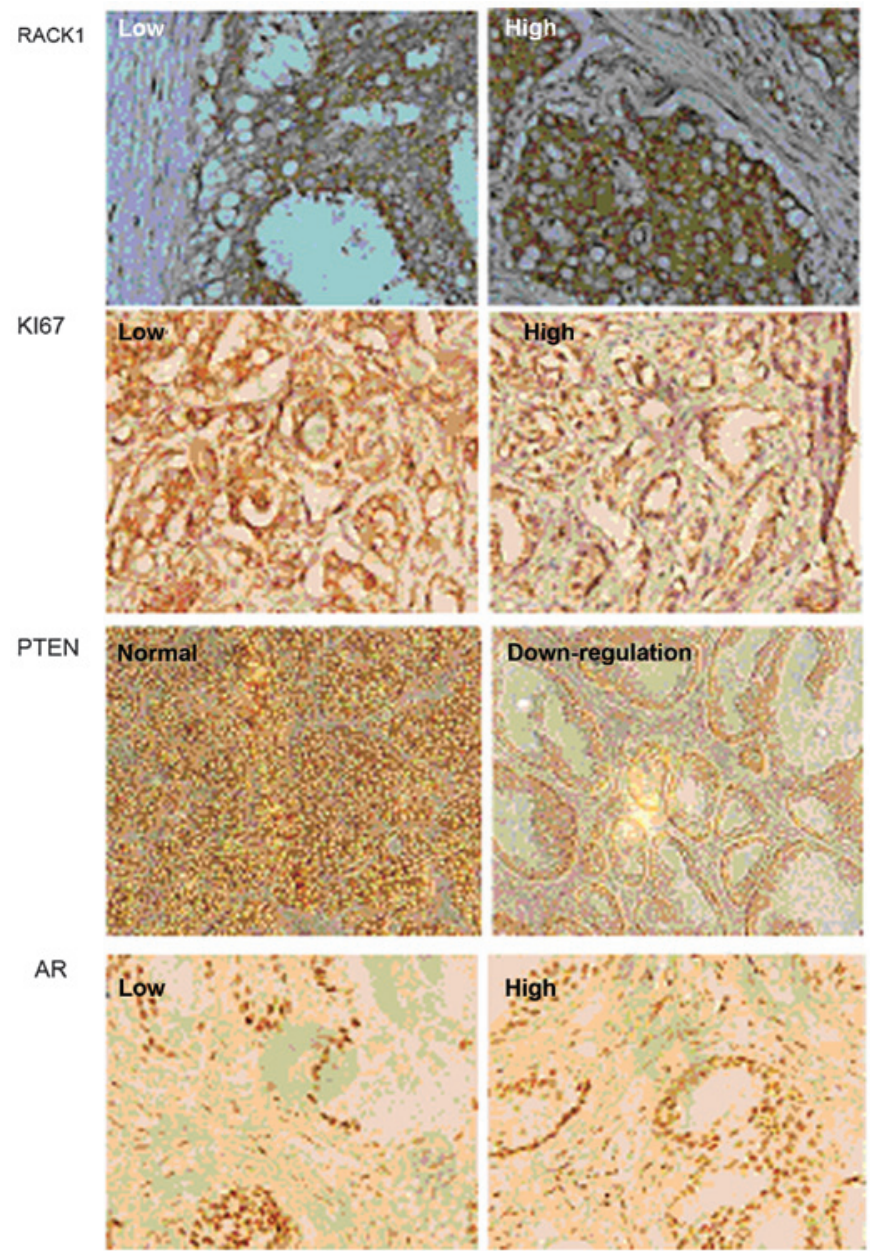

Figure 4. Parallel expression of RACK1, Ki67, PTEN and AR in a welldifferentiated PC tissue sample following immunohistochemical analysis. The immunoreactivity of RACK1, Ki67, PTEN and AR was expressed as a product of the intensity and the proportion of positively stained cells (magnification, x200). AR, androgen receptor; PC, prostate cancer.

was found to be an important predictor of oral squamous cell carcinoma. In the present study, RACK1 staining was found to be closely associated to Ki67 staining (Fig. 4), which is one of the most widely known proliferative predictors in malignant diseases, such as breast carcinoma. Our data indicated that increased RACK1 expression is not only closely related to in vitro cell proliferation, migration and invasion, but also linked to PC cell growth and metastasis in vivo.

The RACK1 pathway is suggested to act through PI3K. The PI3K pathway has also been shown to act through its downstream AKT molecule by regulating various cell functions, including cell transformation, proliferation, apoptosis, angiogenesis and tumor growth. Phosphatase and tensin homolog deleted on chromosome ten (PTEN) is a PI3K inhibitor. PTEN loss or mutation is common in human PC. Inhibition of the PI3K signaling pathway by PTEN inhibits tumor angiogenesis and growth. We have previously demonstrated that AKT is the downstream target of PI3K in controlling angiogenesis and tumor growth, and that PTEN inhibits angiogenesis by regulating the expression of HIF-1 and VEGF expression through AKT activation in PC-3 cells.

AR and PI3K signaling are two of the most important pathways involved in PC. Previous work has shown that there is 
crosstalk between these two pathways (15). AR and PI3K pathways crosstalk in PC cells in vitro as well as in vivo. The PI3K pathway is dominant over AR signaling in PC cells (16-19) (Fig. 4). This should be taken into consideration in future studies on the development of novel therapeutic strategies for PC treatment.

\section{References}

1. Grönberg H: Prostate cancer epidemiology. Lancet 361: 859-864, 2003.

2. Harbeck N, Dettmar P, Thomssen C, et al: Risk-group discrimination in node-negative breast cancer using invasion and proliferation markers: 6-year median follow-up. Br J Cancer 80: 419-426, 1999.

3. Leevers SJ, Vanhaesebroeck B and Waterfield MD: Signalling through phosphoinositide 3-kinases: the lipids take centre stage. Curr Opin Cell Biol 11: 219-225, 1999.

4. Vivanco I and Sawyers CL: The phosphatidylinositol 3-kinase AKT pathway in human cancer. Nat Rev Cancer 2: 489-501, 2002.

5. Fang J, Ding M and Yang L: PI3K/PTEN/AKT signaling regulates prostate tumor angiogenesis. Cell Signal 19: 2487-2497, 2007.

6. Samuels Y, Wang Z, Bardelli A, et al: High frequency of mutations of the PIK3CA gene in human cancers. Science 304: $554,2004$.

7. Broderick DK, Di C, Parrett TJ, et al: Mutations of PIK3CA in anaplastic oligodendrogliomas, high-grade astrocytomas, and medulloblastomas. Cancer Res 64: 5048-5050, 2004.

8. Campbell IG, Russell SE, Choong DY, et al: Mutation of the PIK3CA gene in ovarian and breast cancer. Cancer Res 64: 7678-7681, 2004.
9. Zhao JJ, Gjoerup OV, Subramanian RR, et al: Human mammary epithelial cell transformation through the activation of phosphatidylinositol 3-kinase. Cancer Cell 3: 483-495, 2003.

10. Mao X, Kikani CK, Riojas RA, et al: APPL1 binds to adiponectin receptors and mediates adiponectin signalling and function. Nat Cell Biol 8: 516-523, 2006.

11. Ron D, Chen CH, Caldwell J, et al: Cloning of an intracellular receptor for protein kinase $\mathrm{C}$ : a homolog of the beta subunit of G proteins. Proc Natl Acad Sci USA 91: 839-843, 1994.

12. Berns H, Humar R, Hengerer B, et al: RACK1 is up-regulated in angiogenesis and human carcinomas. FASEB J 14: 2549-2558, 2000 .

13. Han HJ, Russo J, Kohwi Y and Kohwi-Shigematsu T: SATB1 reprogrammes gene expression to promote breast tumor growth and metastasis. Nature 452: 187-193, 2008.

14. Wang Z, Jiang L, Huang C, et al: Comparative proteomics approach to screening of potential diagnostic and therapeutic targets for oral squamous cell carcinoma. Mol Cell Proteomics 7: $1639-1650,2008$

15. Cao XX, Xu JD, Xu JW, et al: RACK1 promotes breast carcinoma proliferation and invasion/metastasis in vitro and in vivo. Breast Cancer Res Treat 123: 375-86, 2010.

16. Mulholland DJ, Tran LM, Li Y, et al: Cell autonomous role of PTEN in regulating castration-resistant prostate cancer growth. Cancer Cell 19: 792-804, 2011.

17. Serrels B, Sandilands E, Serrels A, et al: A complex between FAK, RACK1, and PDE4D5 controls spreading initiation and cancer cell polarity. Curr Biol 20: 1086-1092, 2010.

18. Wang F, Osawa T, Tsuchida R, et al: Downregulation of receptor for activated C-kinase 1 (RACK1) suppresses tumor growth by inhibiting tumor cell proliferation and tumor-associated angiogenesis. Cancer Sci 102: 2007-2013, 2011.

19. Shi Y, Han JJ, Tennakoon JB, et al: Androgens promote prostate cancer cell growth through induction of autophagy. Mol Endocrinol 27: 280-295, 2013. 